\title{
Asenkron Motor Rulman Hatalarının Uzun-Kısa Süreli Bellek Tipi Derin Sinir Ağları ile Otomatik Sınıflandırılması
}

\author{
Rumeysa Hacer K1lıç ${ }^{1}$, Emre Dand1 $1^{2 *}$ \\ ${ }^{1}$ Bilecik Şeyh Edebali Üniversitesi, Lisansüstü Eğitim Enstitüsü, Bilgisayar Mühendisliği Anabilim Dalı, Bilecik, Türkiye, (ORCID: 0000-0002-0768-9133), \\ 3924601@ogrenci.bilecik.edu.tr \\ 2 Bilecik Şeyh Edebali Üniversitesi, Mühendislik Fakültesi, Bilgisayar Mühendisliği Bölümü, Bilecik, Türkiye (ORCID: 0000-0001-6559-1399), \\ emre.dandil@bilecik.edu.tr
}

(Uluslararası Araştırma-Geliştirme ve Tasarım Konferansı - 15-18 Aralık 2021)

(DOI: 10.31590/ejosat.1039896)

ATIF/REFERENCE: Kılıç, R.H., Dandıl, E. (2021). Asenkron Motor Rulman Hatalarının Uzun-Kısa Süreli Bellek Tipi Derin Sinir Ağları ile Otomatik Sınıflandırılması. Avrupa Bilim ve Teknoloji Dergisi, (32), 508-513.

Öz

Endüstride yaygın olarak kullanılan asenkron motorların tercih edilmesinin nedenleri hesaplı, dayanıklı ve güvenilir olmalarıdır. Asenkron motorların iç bilezik, bilye ve dış bilezik kısımlarımda oluşan rulman hataları en sık karşılaşılan hatalardandır. Bu nedenle, asenkron motorlarının çalışmasının verimini arttırmak için rulman hatalarının erken bir aşamada belirlenmesi oldukça önemlidir. Bu çalışmada, Case Western Reserve University (CWRU) rulman veriseti kullanılarak, asenkron motor rulmanlarının iç bilezik, dış bilezik ve bilye bölgelerinde oluşan hataların titreşim verilerinden yararlanarak otomatik sınıflandırılması için iki yönlü uzun-kısa süreli bellek tipi (IY-UKSB) tipi derin sinir ağları tabanlı bir yöntem önerilmektedir. Çalışmada, normal rulman ve hatalı rulmana ait titreşim verileri 128, 256, 512 ve 1024 gibi farklı boyutlarda pencerelere ayrılarak, anlık ferekans ve sprektral entropi ile özellik çıkarımı sonucunda önerilen IY-UKSB ağının performansı değerlendirilmiştir. Çalışmada normal ve hatalı rulman verilerinden oluşturulan veriseti üzerinde farklı pencere genişliklerinde test kümesi üzerinde IY-UKSB ağının doğruluğunun ortalama \%80 civarında kaldığı, buna karşın normal ve hatalı rulman verilerinin sınıflandırılmasında anlık frekans ve spektral entropi ile özellik çıkarımı sonrası IY-UKSB ağının ortalama \%99.28 doğruluk, \%99.72 duyarlılık ve \%97.53 seçicilik skorlarına ulaştığı görülmüştür. Sonuç olarak, önerilen IY-UKSB ağının hatalı ve normal rulman titreşim verilerinin ayrımı için güçlü bir sınıflandırıcı olduğu değerlendirilmiştir.

\section{Automatic Classification of Induction Motor Bearing Faults using Long-Short Term Memory Deep Neural Networks}

\begin{abstract}
The reasons for the preference of induction motors, which are widely used in the industry, are that they are affordable, durable and reliable. Bearing errors in the inner race, ball and outer race parts of induction motors are the most common errors. Therefore, it is very important to detect bearing faults at an early stage in order to increase the efficiency of operation of induction motors. In this study, using Case Western Reserve University (CWRU) bearing dataset, bi-directional long-short-term memory type (Bi-LSTM) deep neural networks are proposed for automatic classification of faults in the inner race, outer race and ball regions of induction motor bearings on vibration data. In the study, the performance of the proposed Bi-LSTM network is evaluated as a result of feature extraction using instantaneous frequency and spectral entropy, by dividing the vibration data of normal bearing and faulty bearing into windows of different sizes such as 128, 256, 512 and 1024. In the study, the accuracy of the Bi-LSTM network for the test set with different window widths on the dataset created from normal and faulty bearing data is $80 \%$ on average, on the other hand, after
\end{abstract}

* Corresponding Author: emre.dandil@bilecik.edu.tr 
feature extraction with instantaneous frequency and spectral entropy in the classification of normal and faulty bearing data, the accuracy of Bi-LSTM network is observed $99.28 \%$ accuracy, 99.72\% sensitivity and 97.53\% specifity scores. As a result, the proposed Bi-LSTM network is considered to be a powerful classifier for the separation of faulty and normal bearing vibration data.

Keywords: Induction motor, Bearing faults, Classification, Bi-directional long-short-term memory (Bi-LSTM).

\section{Giriș}

Asenkron motorlar günlük hayatta ve endüstride yaygın olarak kullanılmaktadır. Güvenirlik, dayanıklılık, etkinlik ve performans asenkron motor uygulamalarındaki bazı önemli hususlardır (Benbouzid, 1999; Zarei, 2012). Asenkron motorlar oldukça güvenilir ve uzun ömürlü olsalar da, bazı hatalara maruz kalabilmektedirler. Bu motorlar yaygın olarak kullanıldığı için, oluşacak hataların önceden sezilmesi ve önlemlerin alınması oldukça önemlidir. Asenkron motorlarda oluşan hatalar sıklıkla titreşim sinyallerinden analiz edilmekle birlikte, akım sinyalleri gibi farklı sinyaller de meydana gelen hataların tespitinde kullanılmaktadır (Benbouzid \& Kliman, 2003).

Asenkron motorlarda hem elektriksel hem de mekanik tipi hatalar maydana gelebilmektedir. Elektriksel hatalar genellikle stator ve rotorda oluşurken, mekanik hatalar çoğunlukla rulmanlar baş göstermektedir. Asenkron motorlarda, rulman hataları oransal olarak en fazla karşılaşılan hata türüdür (Toma vd., 2020; Yeh vd., 2008). Rulmanlarda meydana gelen arızalar endüstride çoğunlukla üretim kalitesini etkilemektedir. Bunun yanında, rulmanlarda meydana gelen arızalar motorda sürekli olarak rezonansa neden olabilmektedir. $\mathrm{Bu}$ nedenle rulmalarda oluşacak hataların tespiti önemli hale gelmektedir. Rulmanlar asenkron motorların hareketli ve bilyeli elemanları olup, iç bilezik, dış bilezik ve kafes bileşenlerinden meydana gelir ve serbest dönme hareketi için rotor ve mili desteklemek için kullanılır (Çalış vd., 2013). Ayrıca rulman asenkron motorlarda sürtünmeyi azaltarak makinenin çalışma performansını arttırmaktadır. Asenkron motorlarda rulman hatalarının yaygın olan mekanik nedenleri arasında sürtünme, motor-yük dengesizliği, uygun olmayan yağlanma bulunmaktadır. Bu tip hatalardan dolayı asenkron motor 1sınıp güç sarfiyatını artırmakta ve/veya asenkron motorda oluşan titreşim nedeniyle performans düşmektedir (Ünsal \& Karakaya, 2015).

Asenkron motorlarda meydana gelebilecek rulman hataların önceden tespit edilebilmesi için literatürde birçok çalışma önerilmiştir. Sinyal analizine dayalı olarak yapılan çalışmalarda, (Mendel $v d ., 2009$ ) rulmanlarda meydana gelebilecek arızaları frekans analizi yöntemiyle tespit etmişlerdir. (Yeşilyurt \& Özdemir, 2015) ise, dengesiz yüke maruz silindirik masuralı rulman hatasını kısa zamanlı fourier dönüşümü kullanarak tespit etmişlerdir. Bir başka çalışmada, (Kompella vd., 2016) rulman hatalarının tespitinde dalgacık dönüşümü ve gürültü giderimi tabanlı bir yaklaşımdan yararlanmışladır. (Demir \& Müştak, 2021) ise çalışmalarında, rulman hatalarını titreşim ve gürültü analizi ile tespit etmeyi önermişlerdir. Çalışmada, titreşim ve gürültü verileri zaman ve frekans düzleminde incelenerek, Hilbert dönüşümü ile zarf analizi yapılmış ve rulman hataları tespit edilmiştir.

Geleneksel makine öğrenmesi yöntemleri ile de asenkron motorlarda oluşan rulman hataların tespiti için önerilen çalışmalar bulunmaktadır. (Konar \& Chattopadhyay, 2011) çalışmalarında rulman hatalarının tespiti için dalgacık dönüşümü ve destek vektör makinası (DVM) ile bir yöntem önermişledir. (Bayram vd., 2013) çalışmalarında rulmanlarda oluşan hatanın boyutunu yapay sinir ağı (YSA) modeli kullanarak belirlemişlerdir. YSA modelinde verileri belirli ağırlıklarla çarpıp gerçek zamanlı olarak giriş olarak verip çeşitli hata boyutlarına sahip rulmanların hata büyüklüğünü sınıflandırmışlardır. Ayrıca gerçek hata değerini ortalama \%2 lik hata oranı ile belirlendiğini gözlemlemişlerdir. Bir diğer çalışmada, (Çalış vd., 2013) çeşitleri rulmanların hataları için titreşim verilerinin zaman ve frekans düzlemindeki genlik değerlerinin analizi ile yapay bağışıklık sistemleri kullanarak rulmanın hatalı olup olmadığını, hatanın yüzde değeri ve seviyesini, titreşim verisinde hangi zaman aralığında hata olduğunun teşhisini uygulamışladır. (Amar vd., 2014) çalışmalarında, YSA kullanarak titreşim spektrumu görüntüleme ile rulman hatası sınıflandırma yaklaşımını sunmuşlardır. Önerilen titreşim spektrumu görüntüleme biçiminde karakteristik titreşimin spektral özelliklerinin görsel temsili güçlendirilerek bir hata sınıflandırcı önerilmiştir. Asenkron motor rulman hatalarının sınıflandırılması için YSA tabanlı önerilen bir başka çalışmada, (Zarei vd., 2014) akıllı bir filtre kullanarak rulman hata tespiti ve YSA ile sınıflandırılması için titreşim analizi yapmışlardır. Doğrudan titreşim sinyali kullanan yaygın yöntemlerden ziyade titreşim sinyalinin filtrelenmiş bileşini kullanıldığında daha iyi sonuçlar elde edildiğini ortaya koymuşlardır. Bir başka çalışmada, (Akkurt \& Arabacı, 2019) sürücüden beslenen asenkron motordaki rulman hatalarının belirlenmesi için akım işaret analizi ve YSA modeli kullanmışlardır. Çalışmalarında rulman hatası sınıflandırılmasını başarılı olarak uygulamışladır. (Hwang vd., 2015) ise çalışmalarında, titreşim sinyali ile asenkron motorlar için DVM tabanlı rulman hatası tespitini önermişledir. Önerilen yöntemde titreşim sinyallerinden arıza ile ilgili frekansların harmoniklerini arıza indeksleri olarak işleyen DVM kullanarak sınıflandırma yapıldığında arıza teşhisinin doğruluunun iyileştiğini göstermişlerdir. Bir diğer çalışmada, (Toma vd., 2020) genetik algoritma ve makine öğrenimli sınıflandırıcı kullanarak asenkron motorlardaki rulman hatalarının tespitini önermişledir. Öncelikle istatistiksel özellikler motor akım verilerinden çıkarılmıştır. Ardından öznitelik sayısını azaltmak ve öznitelik veri tabanından en önemlilerini seçmek için genetik algoritma kullanılmıştır. Rulman hatalarının değerlendirmek için k-en yakın komşu algoritması, karar ağacı ve rastgele orman olmak üzere üç farklı sınıflandırma algoritması bu özellikleri kullanarak eğitilmiş ve test edilmiştir. Deneysel çalışmalar ile üç sınıflandırma yönteminin kombinasyonunun, doğruluğu arttırdı̆̆ 1 ve hesaplama karmaşıklığını azalttığı gözlemlenmiştir.

Bunların yanında, asenkron motorlarda oluşan rulman hatalarının tespitinde, görüntü işleme tekniklerinden de yararlanılmıştır. (Al-Musawi vd., 2020) çalışmalarında, termal görüntü segmentasyonu kullanarak üç fazlı asenkron motorlarda iç bilezik, dış bilezik ve bilye bölgelerindeki rulman hatalarının tespitini gerçekleştirmişlerdir. Çalışmalarında görüntü segmentasyonuna dayalı önerilen HSV renk modelinin motor arızalarını doğru bir şekilde tespit edip tanımlayabildiğini göstermişlerdir.

Son yıllarda, güncel derin öğrenme tabanlı yaklaşımlarda asenkron motor rulman hatalarının tespitinde kullanılmaktadır. (Eren vd., 2019) çalışmalarında asenkron motor rulman hatalarının tespitinde bir boyutlu (1B) evrişimsel sinir ağları (ESA) mimarisinden yararlanmışladır. (Immovilli vd., 2019) 
çalışmalarında motor akım analizi kullanarak uzun-kısa süreli bellek (UKSB) tipi sinir ağları ile rulman hatalarını analiz etmişledir. Bir başka çalışmada, (Jalayer vd., 2021) rulman UKSB tipi sinir ağları, hızlı Fourier dönüşümü ve dalgacık dönüşümü yöntemleri ile hibrit olarak tespit etmişlerdir. (Chen $v d$., 2021) ise çalışmalarında, ESA ve UKSB ile rulman asenkron motor hatalarının tespitini gerçekleştirmişlerdir.

$\mathrm{Bu}$ çalışmada, Case Western Reserve University (CWRU) rulman veriseti (CWRU, 2021) kullanılarak, asenkron motor rulmanlarının iç bilezik, dış bilezik ve bilye bölgelerinde oluşan hataların titreşim verilerinden yararlanarak otomatik sınıflandırılması için UKSB tipi derin sinir ağları tabanlı bir yöntem önerilmektedir. Çalışmada, normal rulman ve farklı boyutlar ve yüklerdeki rulman hatalarına ait iç bilezik, dış bilezik ve bilyeye ait titreşim verileri farklı boyutlarda pencerelere ayrılarak, anlık ferekans ve sprektral entropi ile özellik çıkarımı yapılmıştır. Daha sonra çıkarılan bu özellikler kullanılarak iki yönlü UKSB mimarisi ile hatalı ve hatasız rulman titreşim verilerinin yüksek doğruluk ile sınıflandırılması sağlanmıştır.

\section{Materyal ve Metot}

\subsection{CWRU Rulman Veriseti}

$\mathrm{Bu}$ çalışmada kulllanılan rulman titreşim verileri Case Western Reserve University (CWRU) rulman veriseti (CWRU, 2021)'den alınmıştır. Şekil 1'de görülen deney düzeneğinde rulmanın iç bilezik, bilye ve dış bilezik konumlarına titreşim sensönleri yerleştirilmiştir. Ayrıca dış bilezik üzerinden üç farklı konumdan veriler toplanmıştır. Verisetinde hem normal (sağlıklı) çalışan asenkron motor rulmanı üzerinde, hem de yapay olarak aşındırılmış asenkron motor üzerinde veri toplama süreci tekrar edilmiştir.

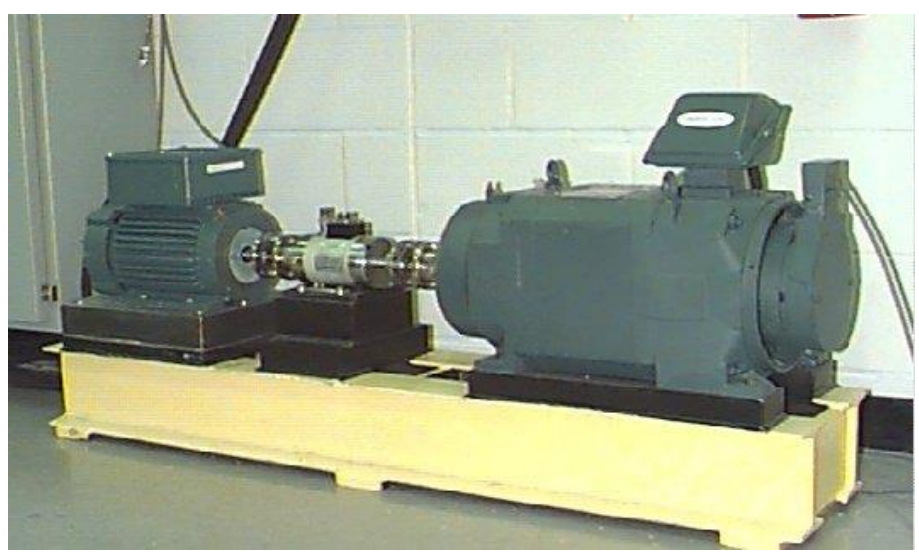

Şekil 1.CWRU verisetinde rulman titreşim verilerinin alındı $\breve{l}$ tümleşik deney düzene $\breve{i}$

Tablo 1. CWRU rulman verisetinde deneysel çalışmalar için kullanılan üç fazlı asenkron motorun özellikleri

\begin{tabular}{l|l}
\hline Özellik & Değer \\
\hline Gücü (Hp) & 2 \\
\hline Gerilim (V) & $208-230$ \\
\hline Akım (A) & $1.5-3.0$ \\
\hline Frekans (Hz) & 60 \\
\hline Çalışma ortamı sıcaklığı $\left(C^{0}\right)$ & 40 \\
\hline Ağırık (kg) & 38.00 \\
\hline Devir sayısı (d/dk) & $1800(1720-1797)$ \\
\hline
\end{tabular}

CWRU verisetinde hem normal/sağlıklı hem de hatalı rulman verileri bulunmaktadır. Deneysel düzenekte $2 \mathrm{hp}$ gücündeki ve detayları Tablo 1'de sunulan Reliance Electric firmasının IQPreAlert üç fazlı asenkron motoru kullanılmıştır. Asenkron motorun dönüș hızının dakikada 1725, 1750, 1772 ve 1769 devir olduğu durumlarda veriler kaydedilmiştir. Bu devirlerdeyken asenkron motor sirasıla 3, 2, 1 ve 0 yük durumundadır. CWRU verisetinde elektrik motorunun sağlıklı titreşim verileri kaydedildikten sonra rulmanda farklı boyutlarda (0.007, 0.014, 0.021 ve 0.028 inç) yapay olarak aşındırma yapılarak bilye, iç bilezik ve diş bilezik konumlarından sensörler yardımıyla asenkron motorun titreşim verileri kaydedilmiştir.

CWRU rulman verisetinde asenkron motorun sürücü sonuna ve fan sonuna sensörler yerleştirilerek, buralardaki rulmanlara ait titreşim verileri toplanmıştır. Bu çalışmada ise sadece sürücü sonundan alınan rulman titreşim verileri kullanılmıştır. Normal veriler asenkron motorun rulmanın sürücü sonunda tek bir noktadan toplanmıştır. Hatalı çalışma sırasında asenkron motorun rulamının sürücü sonundan 5 farklı noktadan örnekler alınmıştır. Bunlar titreşim verileri iç bilezik, bilye ve dış bilezik için üç ayrı konumdan alınmıştır. Hatalı çalışan asenkron motorun sürücü sonundaki rulmana ait titreşim verileri her 12000 saniyede örnek içerecek şekilde, $5000 \mathrm{~Hz}, 1 \mathrm{v} / \mathrm{g}$ özelliklerine sahip titreşim ölçer aracılığıyla toplanmıştır. Toplanan veriler yükssek bant genişliği ve $5 \mathrm{kHz}$ kesme frekansı olan bir açlak geçiren filtre ile sınırlandırılmıştır. Asenkron motorun rulmanından elde edilen tüm titreşim verileri .mat uzantılı olarak Matlab dosyası şeklinde kaydedilmiştir. Hatalı asenkron motorun ve hatasız asenkron mototrun özellikleri aşağıdaki çizelde verilmiştir. Normal rulman verileri $0,1,2$ ve 3 yük durumlarında kaydedilmiş olup, $12 \mathrm{~K}$ sürücü sonu hatalı rulman titreșim verilerinin dağılımı Tablo 2'de gösterilmiștir. Tabloda + ile kullanılabilir durumdaki titreşim verileri, - ile kullanılamaz durumdaki titreşim verilerini temsil edilmiştir. Tabloda dış bilezikten alınan titreşim verilerinin saat@12:00, saat@3:00 ve saat@6:00 konumlarından alındığ ifade edilmiştir.

Tablo 2. $12 \mathrm{~K}$ sürücü sonu hatalı rulman titreşim verilerinin dă̆ılımı

\begin{tabular}{|c|c|c|c|c|c|c|}
\hline \multirow{2}{*}{$\begin{array}{l}\text { Hatanın } \\
\text { Büyükllüğü } \\
\text { (inç) }\end{array}$} & \multirow[b]{2}{*}{ Yük } & \multirow{2}{*}{$\begin{array}{c}\text { İç } \\
\text { Bilezik }\end{array}$} & \multirow[b]{2}{*}{ Bilye } & \multicolumn{3}{|c|}{ Dıș Bilezik } \\
\hline & & & & @12:00 & (a) 3:00 & (a)6:00 \\
\hline \multirow{4}{*}{$0,007 "$} & 0 & + & + & + & + & + \\
\hline & 1 & + & + & + & + & + \\
\hline & 2 & + & + & + & + & + \\
\hline & 3 & + & + & + & + & + \\
\hline \multirow{4}{*}{0,014 " } & 0 & + & + & + & - & - \\
\hline & 1 & + & + & + & - & - \\
\hline & 2 & + & + & + & - & - \\
\hline & 3 & + & + & + & - & - \\
\hline \multirow{4}{*}{$0,021 ”$} & 0 & + & + & + & + & + \\
\hline & 1 & + & + & + & + & + \\
\hline & 2 & + & + & + & + & + \\
\hline & 3 & + & + & + & + & + \\
\hline \multirow{4}{*}{$0,028^{\prime \prime}$} & 0 & + & + & - & - & - \\
\hline & 1 & + & + & - & - & - \\
\hline & 2 & + & + & - & - & - \\
\hline & 3 & + & + & - & - & - \\
\hline
\end{tabular}




\subsection{Uzun-kısa süreli bellek (UKSB) tipi sinir ağları}

Uzun kısa süreli bellek (UKSB) ağı ilk olarak Sepp Hochretier ve Jürgen Schminhuber tarafından (Hochreiter \& Schmidhuber, 1997) ortaya atılmıştır. UKSB derin öğrenme alanında kullanılan ve zaman adımları arasındaki uzun zamanlı bağımlılıkları öğrenebilen bir tür tekrarlayan sinir ağ mimarisidir. UKSB ağlarının temel bileşenleri giriş katmanı ve UKSB katmanıdır. Giriş katmanında dizi veya zaman serileri şeklindeki verilerin ağa sunulur. UKSB katmanı ise dizi verilerinin zaman adımları arasındaki uzun vadeli bağımlılıklarını öğrenir.

UKSB ağları zaman serisi verilerine dayanarak sınıflandırmak, işlemek ve tahmin yapmak için uygun bir yapıya sahiptir. Aynı zamanda, UKSB'ler yalnızca anlık veriyi değil dizi verilerinide işleyebilir. UKSB ağlarından konuşma tanıma (Sak $v d$., 2014), ses tanıma (Graves $v d$., 2013), el yazısı tanıma (Stuner $v d ., 2020)$ gibi birçok uygulamanın gerçeklenmesinde yararlanılmıştır.

Hafiza bloğu sayesinde UKSB hücreleri, önemi yüksek olan bilgiyi geri yayılımda kullanılırken önemi düşük olanları unutarak çalışmaktadır. İki yönlü uzun kısa süreli bellek (IYUKSB) ağları aynı süreçte iki UKSB ağının eğitilmesi ile prensibi ile çalışmaktadırlar (Schuster \& Paliwal, 1997). Ağın girdisi, aynen ve zamana göre tersi alınmış hali ile kullanılmaktadır. $\mathrm{Bu}$ çalışma şeklinin avantajı, ă̆ geçmiş hakkındaki bilgilerin yanında gelecek hakkında bilgileri de tutmuş olmaktadır. Hafıza bloğunun yapısı belli kapılardan oluşmaktar. Unutma kapısı, giriş kapısı, güncelleme kapısı ve çıkış kapısı ağın yapısındaki kapılardır. Uzun kısa süreli bellek ağları modeli için eşitlikler Denklem (1), Denklem (2), Denklem (3), Denklem (4), Denklem (5) ve Denklem (6)'da verilmişstir (Dand1l \& Karaca, 2020):

$$
\begin{aligned}
& f_{t}=\sigma\left(w_{f} \cdot\left[h_{t-1}, x_{t}\right]+b_{f}\right) \\
& i_{t}=\sigma\left(w_{i} \cdot\left[h_{t-1}, x_{t}\right]+b_{i}\right) \\
& g_{t}=\tanh \left(w_{g} \cdot\left[h_{t-1}, x_{t}\right]+b_{g}\right) \\
& c_{t}=f_{t} * c_{t-1}+i_{t} * g_{t} \\
& o_{t}=\sigma\left(w_{o} \cdot\left[h_{t-1}, x_{t}\right]+b_{o}\right) \\
& h_{t}=o_{t} * \tanh \left(c_{t}\right)
\end{aligned}
$$

Denklem (1) unutma kapısının çıktısını, Denklem (2) giriş kapısının çıktısını, Denklem (3) güncelleme kapısının çıktısını, Denklem (4) hafıza hücresini ve Denklem (5) çıkış kapısının çıktısını temsil etmektedir. $t$ - 1 bir önceki zaman adımını, gizlilik durumu ise $h_{t}-1$ ile gösterilmiştir. Bu denklemlerde $w$, ağırlık matrislerini, $b$ ise bias vektörünü ifade etmektedir. Denklem (6) hücrenin gizlilik durumu, $\sigma$ sigmoid aktivasyon fonksiyonu için kullanılmaktadır (Dandıl \& Karaca, 2020).

\section{Araştırma Sonuçları ve Tartışma}

Bu çalışmada, CWRU veri tabanından alınan sağlıklı çalışan asenkron motorun rulmanına ait titreşim verileri ve yapay olarak aşındırılan hatalı çalışan asenkron motorun rulmanına ait titreşim verilerinin IY-UKSB derin sinir ağları ile otomatik sınıflandırılması için bir model oluşturulmuştur. Bu çalışmada yürütülen deneysel analizler, Intel(R) Core (TM) i7-9750H CPU (a) 2.60GHz 2.60 GHz işlemci, 16GB bellek ve NVDIA Geforce GTX 1160 Ti ekran kartına sahip Windows 10 işletim sistemiyle çalışan bir bilgisayarda yapılmıştır. Deneysel çalışmalar kapsamındaki uygulamalar MATLAB programlama dili ortamında geliştirilmiştir. IY-UKSB ağının mimarisinde sırasıyla bir giriş katmanı (sequenceInputLayer), bir IY-UKSB katmanı (bilstmLayer), bir tam bağlı katman (fullyConnectedLayer), bir softmax katmanı ve son olarak bir sinıflandırma katmanı (classificationLayer) bulunmaktadır.

CWRU rulman verisetinde asenkron motorun sürücü sonuna ve fan sonuna sensörler yerleştirilerek rulmanlara ait titreşim verileri toplanmıştır. Normal rulmana ait veriler sürücü sonunda tek bir noktadan toplanmıştır. Bunun yanında, bu çalışmada ise sadece $12 \mathrm{~K}$ sürücü sonu hatalı rulman titreşim verileri kullanılmıştır. Hatalı çalışan asenkron motorun sürücü sonundaki rulmana ait titreşim verileri her 12000 saniyede $(12 \mathrm{~K})$ örnek içerecek şekilde, $5000 \mathrm{~Hz}, 1 \mathrm{v} / \mathrm{g}$ özelliklerine sahip titreşim ölçer (akselerometre) aracılığıyla toplanmıştır.

Çalışmada, deneysel analizler için normal ve hatalı rulmanlara ait her bir veri 128, 256, 512 ve 1024 'lük pencerelere bölünerek matrislere ayrılmıştır. Görselleştirilen çalışmada deneysel analizler için, sağlıklı asenkron motor Yük 1 durumunda iken ve yine Yük 1 durumunda iken hata büyüklüğü 0.014 " olan asenkron motorun iç bileziğinden elde edilen hatalı rulman titreşim verileri kullanılmıştır. Şekil 2'de motorun Yük 1 durumunda normal rulman verileri (Şekil 2(a)) ile yine motorun Yük 1 durumunda 0.014 " hata büyüklüğünde iç bilezikten (Şekil 2(b)) 512 pencere boyutunda alınmış titreşim verilerine ait sinyalleri gösterilmiştir.

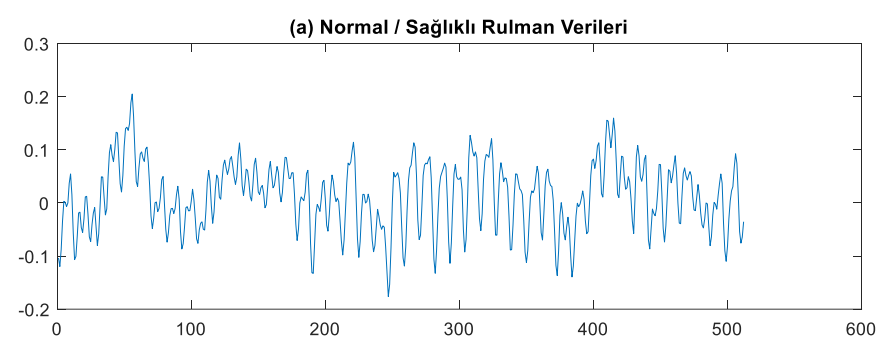

(b) İç Bilezik Hatalı Rulman Veriler

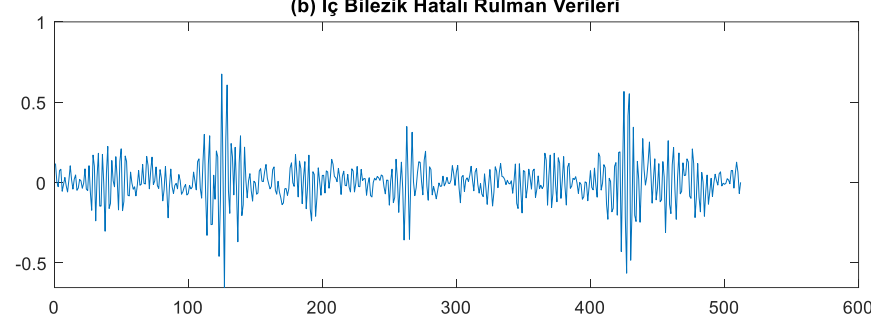

Şekil 2. (a) Normal rulman verileri ve (b) hatall rulmana ait iç bilezikten alınan 512 pencere genişliğinde tireşim sinyalleri

Deneysel çalışmalarda öncelikle veriler IY-UKSB ağı için uygun hale getirilmiştir. Her bir deneysel analizde, verilerin \%70’i ağın eğitim aşaması için, kalan \%30’u ise test aşaması için kullanılmıştır. Ayrıca, ağın performansını tam olarak ölçebilmek için, veri arttırımı yapılarak eğitim verileri 5 katına kadar çoğaltılmıştır. Test verileri için ise herhangi bir veri artırma prosedürü uygulanmamıştır. IY-UKSB ağının mimarisinde epok sayısı 10 ve öğrenme katsayısı 0.01 olarak belirlenmiştir. Şekil 3'te 1024 pencere boyutunda normal ve hatalı rulman verilerinin snıflandırılması için oluşturulan IYUKSB ağının eğitim aşamasında elde edilen doğruluk ve yaklaşık kayıp grafikleri gösterilmiştir. 


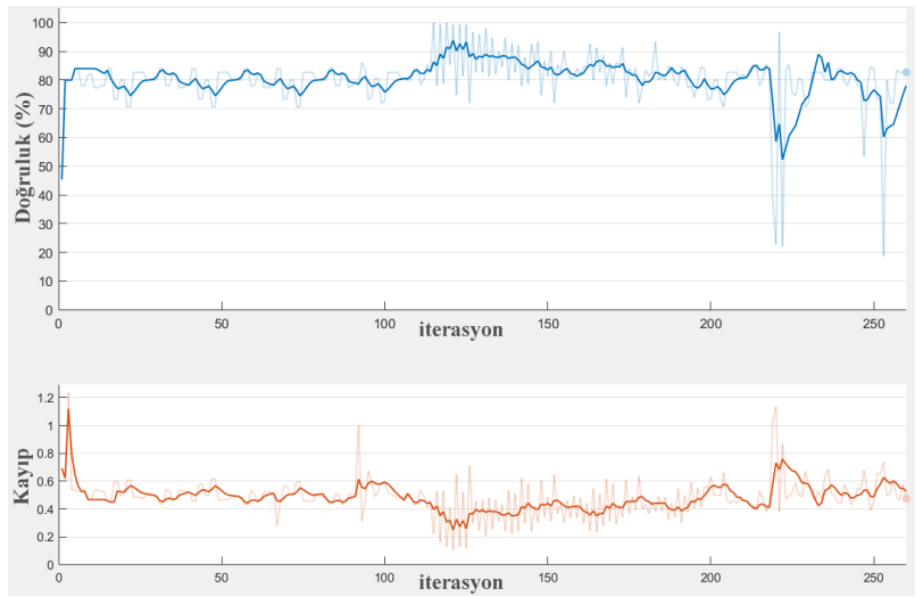

Şekil 3.1024 pencere boyutunda normal ve hatalı rulman verilerinin snıflandırılması için oluşturulan IY-UKSB ă̆ının eğitim aşamasında elde edilen doğruluk ve yaklaşık kayıp grafikleri

Çalışmamızda önerilen IY-UKSB ağının normal ve hatalı rulman verilerini sınıflandırmadaki başarımı, Denklem (7), Denklem (8) ve Denklem (9)'da sunulan ve karmaşlıklık matrislerindeki Doğru Pozitif (DP), Yanlış Negatif (FN), Doğru Negatif (DN), Yanlış Pozitif (YP) değerlerine bağlı olarak hesaplanan Doğruluk (DG), Duyarlılık (DY) ve Seçicilik (SC) performans kriterleri ile ölçülmüştür.

$$
\begin{aligned}
& D G=\frac{D P+D N}{D P+D N+Y P+Y N} \\
& D Y=\frac{D P}{D P+Y N} \\
& S C=\frac{D N}{D N+Y P}
\end{aligned}
$$

\begin{tabular}{|c|c|c|c|c|c|c|c|}
\hline \multicolumn{8}{|c|}{ Özellik çıkarımı olmadan IY-UKSB ağııın performansı } \\
\hline $\begin{array}{l}\text { Pencere } \\
\text { boyutu }\end{array}$ & DP & YP & $\mathbf{Y N}$ & DN & $\begin{array}{l}\text { DG } \\
(\%)\end{array}$ & $\begin{array}{l}\text { DY } \\
(\%)\end{array}$ & $\begin{array}{l}\text { SC } \\
\text { (\%) }\end{array}$ \\
\hline 128 & 1120 & 200 & 14 & 85 & 84.92 & 98.77 & 29.82 \\
\hline 256 & 554 & 113 & 13 & 30 & 82.25 & 97.71 & 20.98 \\
\hline 512 & 272 & 53 & 12 & 18 & 81.69 & 95.77 & 28.35 \\
\hline 1024 & 134 & 27 & 8 & 8 & 80.23 & 94.37 & 22.86 \\
\hline & & & & & 82.27 & 96.66 & 25.50 \\
\hline
\end{tabular}

Tablo 3. Özellik çıkarımı öncesi ve özellik çıkarımı sonrası farklı pencere boyutlarında IY-UKSB ă̆ının başarımı

Anlık frekans ve spektral entropi ile özellik çıkarımı sonrası IYUKSB ă̆ının performanst

\begin{tabular}{llllllll}
\hline $\begin{array}{l}\text { Pencere } \\
\text { boyutu }\end{array}$ & DP & YP & YN & DN & $\begin{array}{l}\text { DG } \\
(\%)\end{array}$ & $\begin{array}{l}\text { DY } \\
(\%)\end{array}$ & $\begin{array}{l}\text { SC } \\
(\%)\end{array}$ \\
\hline & & & & & & & \\
128 & 1133 & 4 & 1 & 281 & 99.65 & 99.91 & 98.60 \\
256 & 565 & 4 & 2 & 139 & 99.15 & 99.65 & 97.20 \\
512 & 284 & 2 & 0 & 69 & 99.44 & 100 & 97.18 \\
1024 & 141 & 1 & 1 & 34 & 98.87 & 99.30 & 97.14 \\
\hline \multicolumn{7}{r}{} \\
\hline
\end{tabular}

Çalışmada normal ve hatalı rulman verilerinden oluşturulan veriseti üzerinde $128,256,512$ ve 1024 pencere genişliklerinde eğitim aşaması tamamlandıktan sonra, test kümesi üzerinde IYUKSB ağının performansı analiz edilmiştir. Ancak yapılan deneysel çalışmalarda IY-UKSB ağının doğruluğunun ortalama $\% 80$ civarında kaldığı görülmüştür. $\mathrm{Bu}$ aşamada, doğruluğun düşük olması nedeniyle normal ve hatalı rulman titreşim verilerinde anlık frekans ve spektral entropi ile özellik çıkarımı yapılmıştır. Sinyallerden özellik çıkarımı yapıldıktan sonra, IYe-ISSN: 2148-2683
UKSB ağı yeniden eğitilmiş ve test kümesi üzerinde yeniden performan kriterleri ölçülmüştür. $\mathrm{Bu}$ durumda hatalı ve normal rulman verilerinin IY-UKSB ağı ile sinıflandırılmasında doğruluk olukça yükselmiştir. Tablo 3 'te özellik çıkarımı öncesi ve özellik çıkarımı sonrası farklı pencere boyutlarında IY-UKSB ağının başarımı gösterilmiştir. Buradan, normal ve hatalı rulman verilerinin sınıflandirılmasında anlık frekans ve spektral entropi ile özellik çıkarımı sonrası IY-UKSB ağının ortalama \%99.28 DG, \%99.72 DY ve \%97.53 SC skorlarına ulaştığ görülmektedir.

\section{Sonuçlar ve Öneriler}

$\mathrm{Bu}$ çalışmada asenkron motorun rulman hatasının tespiti ve sınıflandırılması için IY-UKSB ağı kullanılarak ve anlık frekans ve spektral entropi ile özellik çıkarımı tabanlı bir yöntem önerilmiştir. Deneysel çalışmalarda, CWRU verisetindeki şeşitli büyüklüklerde ve yapay olarak aşındırılmış üç fazlı asenkron motorun farklı yüklerdeki titreşim verileri kullanılmıştır. Çalışmada normal ve hatalı rulman verilerinden oluşturulan veriseti üzerinde 128, 256, 512 ve 1024 pencere genişliklerinde test kümesi üzerinde IY-UKSB ağının doğruluğunun ortalama \%80 civarında kaldığ 1 , ancak normal ve hatalı rulman verilerinin sinıflandırılmasında anlık frekans ve spektral entropi ile özellik çıkarımı sonrası IY-UKSB ağının ortalama \%99.28 DG, \%99.72 DY ve \%97.53 SC skorlarına ulaştığı görülmüştür. Bu çalışmada anlık frekans ve spektral entropi ile özellik çıkarımı sonrası IYUKSB ağının hatalı ve normal rulman titreşim verilerinin sınıflandırılmasında güçlü bir ayrım imkânı sağladığı gözlemlenmiştir. Ayrıca, farklı boyuttaki pencere büyüklüklerinin $(128,256,512$ ve 1024) sınıflandırma başarısına aşırı bir etkisi gözlenmemiştir. Sonraki çalışmalarda, hatalı rulmanların farklı konum, yük ve hata büyüklüklerine göre alınan titreşim verileri üzerinde farklı sınıflandırma yöntemlerinin karşılaştırılması planlanmaktadır.

\section{Teşekkür}

Çalışmanın yazarları çalışmada kullanılan normal ve hatalı rulman titreşim verilerini sağlayan CWRU'ya teşekkürlerini sunmaktadir.

\section{Kaynakça}

Akkurt, İ., \& Arabacı, H. (2019). Sürücüden Beslenen Asenkron Motorlarda Rulman Arızalarının Stator Akımı Kullanarak Tespiti. Uluslararası Doğu Anadolu Fen Mühendislik ve Tasarım Dergisi, 1(2), 122-134.

Al-Musawi, A. K., Anayi, F., \& Packianather, M. (2020). Threephase induction motor fault detection based on thermal image segmentation. Infrared Physics \& Technology, $104,103140$.

Amar, M., Gondal, I., \& Wilson, C. (2014). Vibration spectrum imaging: A novel bearing fault classification approach. IEEE transactions on Industrial Electronics, 62(1), 494-502.

Bayram, S., Kaplan, K., Kuncan, M., \& Ertunç, H. M. (2013, 2628 Eylül 2013). Bilyeli rulmanlarda zaman uzayında istatistiksel öznitelik çıkarımı ve yapay sinir ăgları metodu ile hata boyutunun kestirimi. Paper presented at the Otomatik Kontrol Ulusal Toplantis (TOK2013), Malatya. pp. 986-991. 
Benbouzid, M. (1999). Bibliography on induction motors faults detection and diagnosis. IEEE Transactions on Energy Conversion, 14(4), 1065-1074.

Benbouzid, M., \& Kliman, G. B. (2003). What stator current processing-based technique to use for induction motor rotor faults diagnosis? IEEE Transactions on Energy Conversion, 18(2), 238-244.

Chen, X., Zhang, B., \& Gao, D. (2021). Bearing fault diagnosis base on multi-scale CNN and LSTM model. Journal of Intelligent Manufacturing, 32, 971-987.

CWRU. (2021). Case Western Reserve University Bearing Data Center. Available online: https://engineering.case.edu/bearingdatacenter

Çalış, H., Cakir, A., \& Dandil, E. (2013). Artificial immunitybased induction motor bearing fault diagnosis. Turkish Journal of Electrical Engineering and Computer Science, 21(1), 1-25.

Dand1l, E., \& Karaca, S. (2020). MR Spektroskopi Sinyalleri Kullanılarak LSTM Derin Sinir Ağları ile Beyinde Sahte Tümörlerin Tespiti. Avrupa Bilim ve Teknoloji Dergisi, 426-433.

Demir, H. G., \& Müştak, O. (2021). Rulman Hasarlarının Titreşim ve Gürültü Analizi ile Tespiti. Avrupa Bilim ve Teknoloji Dergisi(25), 571-581.

Eren, L., Ince, T., \& Kiranyaz, S. (2019). A generic intelligent bearing fault diagnosis system using compact adaptive 1D CNN classifier. Journal of Signal Processing Systems, 91(2), 179-189.

Graves, A., Mohamed, A.-r., \& Hinton, G. (2013). Speech recognition with deep recurrent neural networks. Paper presented at the 2013 IEEE international conference on acoustics, speech and signal processing. pp. 6645-6649.

Hochreiter, S., \& Schmidhuber, J. (1997). Long short-term memory. Neural computation, 9(8), 1735-1780.

Hwang, D.-H., Youn, Y.-W., Sun, J.-H., Choi, K.-H., Lee, J.-H., \& Kim, Y.-H. (2015). Support vector machine based bearing fault diagnosis for induction motors using vibration signals. Journal of Electrical Engineering and Technology, 10(4), 1558-1565.

Immovilli, F., Lippi, M., \& Cocconcelli, M. (2019). Automated bearing fault detection via long short-term memory networks. Paper presented at the 2019 IEEE 12th International Symposium on Diagnostics for Electrical Machines, Power Electronics and Drives (SDEMPED). pp. 452-458.

Jalayer, M., Orsenigo, C., \& Vercellis, C. (2021). Fault detection and diagnosis for rotating machinery: A model based on convolutional LSTM, Fast Fourier and continuous wavelet transforms. Computers in Industry, 125, 103378.

Kompella, K. D., Mannam, V. G. R., \& Rayapudi, S. R. (2016). DWT based bearing fault detection in induction motor using noise cancellation. Journal of Electrical Systems and Information Technology, 3(3), 411-427.

Konar, P., \& Chattopadhyay, P. (2011). Bearing fault detection of induction motor using wavelet and Support Vector Machines (SVMs). Applied Soft Computing, 11(6), 4203-4211.

Mendel, E., Rauber, T. W., Varejão, F. M., \& Batista, R. J. (2009). Rolling element bearing fault diagnosis in rotating machines of oil extraction rigs. Paper presented at the 2009 17th European Signal Processing Conference. pp. 1602-1606.
Sak, H., Senior, A. W., \& Beaufays, F. (2014). Long short-term memory recurrent neural network architectures for large scale acoustic modeling. Available online: https://storage.googleapis.com/pub-tools-publicpublication-data/pdf/43905.pdf

Schuster, M., \& Paliwal, K. K. (1997). Bidirectional recurrent neural networks. IEEE transactions on Signal Processing, 45(11), 2673-2681.

Stuner, B., Chatelain, C., \& Paquet, T. (2020). Handwriting recognition using cohort of LSTM and lexicon verification with extremely large lexicon. Multimedia Tools and Applications, 79(45), 34407-34427.

Toma, R. N., Prosvirin, A. E., \& Kim, J.-M. (2020). Bearing fault diagnosis of induction motors using a genetic algorithm and machine learning classifiers. Sensors, 20(7), 1884.

Ünsal, A., \& Karakaya, O. (2015). ASENKRON MOTOR ROTOR ARIZALARININ ANALIZİ. Journal of Science and Technology of Dumlupinar University(034), 69-86.

Yeh, C.-C., Sizov, G. Y., Sayed-Ahmed, A., Demerdash, N. A., Povinelli, R. J., Yaz, E. E., \& Ionel, D. M. (2008). A reconfigurable motor for experimental emulation of stator winding interturn and broken bar faults in polyphase induction machines. IEEE Transactions on Energy Conversion, 23(4), 1005-1014.

Yeşilyurt, İ., \& Özdemir, Ö. (2015). Dengesiz Yüke Maruz Silindirik Masuralı Rulman Arızasının Kısa Zamanlı Fourier Dönüşümü Yardımıyla Belirlenmesi. Uluslararası Katılımlı 17. Makina Teorisi Sempozyumu, 17, 1-8.

Zarei, J. (2012). Induction motors bearing fault detection using pattern recognition techniques. Expert systems with Applications, 39(1), 68-73.

Zarei, J., Tajeddini, M. A., \& Karimi, H. R. (2014). Vibration analysis for bearing fault detection and classification using an intelligent filter. Mechatronics, 24(2), 151157. 